\title{
In silico approach of finding inhibitor of Nedd8-activating enzymes for cancers
}

\author{
P. Kumar*, S. Piramanayagam \\ Department of Bioinformatics, Bharathiar University, Coimbatore, India \\ *e-mail:sirajane@gmail.com
}

Key words: NEDD8, pharmacophore, docking, Schrodinger, Symyx draw

Motivation and Aim: Cancer normally refers to diseases in which abnormal cells will divide without control and it can able to invade the other tissues. NEDD8 (Neural Precursor Cell Expressed Developmentally Downregulated Protein 8)-Activating Enzyme is an essential component of the NEDD8 conjugation pathway that controls the activity of the cullin RING subtype of ubiquitin ligases, which regulates the turnover of a subset of proteins upstream of the proteosome. This work is focused on identifying the inhibitors for NEDD8 with already identified first-class inhibitor MLN4924 [1].

Methods and Algorithms: The pharmacophore studies and ADME-Tox analysis was used to screen the compounds to find out the optimal lead molecules. The molecular docking studies was carried out to find out the molecular binding affinity of protein and ligand molecules.

Results: In the first phase 200 ligands were generated and evaluated by using Ramachandran plot. ADME-Tox was carried out and found 100 molecules had toxic substances. Finally 18 lead molecules was generated as a potent ligands with druggable properties.

Conclusion: This work was carried out based on Fragment Based Drug Design. Based on the Pharmacophore analysis and ADMET studies, the optimal lead molecules were identified. These ligands were compared with already available anti cancer drugs. The NEDD8-Activating enzyme lead molecules are generated by using Fragment Based Drug Design and it's active against angiogenesis

Acknowledgements: I acknowledge UGC-RGNF and DBT-BIF, New Delhi for providing computational facilities to carried out this research work.

\section{References}

1. Qiang Xu et al. (2017) MLN4924 neddylation inhibitor promotes cell death in paclitaxel-resistant human lung adenocarcinoma cells. Oncol. Lett. 15(1):515-521. 